\title{
Tolerability and efficacy of a combination of paracetamol and caffeine in the treatment of tension-type headache: a randomised, double-blind, double-dummy, cross-over study versus placebo and naproxen sodium
}

\author{
Luigi Alberto Pini - Enrico Del Bene - Giorgio Zanchin • Paola Sarchielli • \\ Girolamo Di Trapani · Maria Pia Prudenzano · Giovanni LaPegna • \\ Lidia Savi · Giorgio Di Loreto · Paolo Dionisio · Franco Granella
}

Received: 29 June 2008/Accepted: 2 September 2008/Published online: 25 September 2008 (C) Springer-Verlag 2008

\begin{abstract}
The main aim of this study was to confirm in an Italian population affected by tension-type headache (TTH) the good profile of safety and tolerability of the combination paracetamol 1,000 mg-caffeine $130 \mathrm{mg}$ (PCF) observed in previous studies, by a comparison with naproxen sodium $550 \mathrm{mg}$ (NAP) and placebo (PLA). A secondary objective
\end{abstract}

\author{
L. A. Pini \\ Centro Cefalee, Azienda Ospedaliera Universitaria, \\ Modena, Italy \\ E. Del Bene \\ Centro Cefalee, Azienda Ospedaliera Universitaria Careggi, \\ Florence, Italy \\ G. Zanchin \\ Azienda Ospedaliera Universitaria, Padua, Italy \\ P. Sarchielli \\ Policlinico Monteluce, Perugia, Italy \\ G. Di Trapani \\ Università del Sacro Cuore, Policlinico Gemelli, Rome, Italy
}

M. P. Prudenzano

Azienda Ospedaliera Policlinico Consorziale, Bari, Italy

G. LaPegna

Azienda USL 2, Caltanissetta, Italy

L. Savi

Ospedale S. Giovanni Battista Molinette, Turin, Italy

G. Di Loreto · P. Dionisio

Direzione Medica Centrale, Angelini Farmaceutici,

ACRAF SpA, Rome, Italy

F. Granella $(\bowtie)$

Dipartimento di Neuroscienze, Università di Parma,

Via Gramsci, 14, 43100 Parma, Italy

e-mail: franco.granella@unipr.it was to assess the efficacy of PCF in the acute treatment of TTH. This was a multicentre, randomised, double-blind, double-dummy, crossover, placebo-controlled trial. Tolerability was assessed by recording adverse events by the patient in the 4-h post-dose treatment. To assess the efficacy, the sum of pain intensity differences (SPID) and the total pain relief (TOTPAR) were calculated. Comparing PCF and NAP and PCF and PLA for tolerability, the difference was nonsignificant but the result regarding noninferiority was inconclusive, whilst NAP was noninferior to PLA. As regards SPID and TOTPAR, both PCF and NAP were better than placebo $(P<0.05)$, but not significantly different from each other. In conclusion, PCF was well-tolerated and effective in the treatment of acute TTH.

Keywords Tension-type headache - Acute treatment . Paracetamol - Caffeine - Paracetamol-caffeine combination $\cdot$ Naproxen sodium

\section{Introduction}

Tension-type headache (TTH) is the most prevalent primary headache with a worldwide lifetime prevalence of $46 \%$ [1]. In Europe the lifetime prevalence of TTH seems to be even higher: it was as high as $86 \%$ in a populationbased study in Denmark [2]. Even if in that study most of the patients had episodic infrequent TTH (1 day or less per month), nevertheless 24-37\% had TTH several times a month and $10 \%$ had TTH weekly. Due to its high prevalence, TTH carries a great (and still partially unexplored) burden for the affected individuals and the society. The results of two Danish studies have shown that the number of work days missed in the population owing to TTH was three times higher than the number for migraine $[3,4]$; and 
a US study has also found that absenteeism due to TTH is considerable [5]. Under the assumption that the indirect costs of TTH far outweigh the direct costs, we can therefore speculate that the cost of TTH is greater than the cost of migraine $[1,6]$.

Given the high prevalence and costs of TTH, it is of paramount importance to have safe, well-tolerated, and effective drugs to treat TTH at our disposal. Lacking specific drugs, as triptans are for the acute treatment of migraine, NSAIDs are commonly employed [7]. Some NSAIDs have been proved to be superior to placebo in the acute treatment of TTH [8]. However, gastric irritation and occasionally ulceration may complicate treatment with aspirin or other NSAIDs, even when they are used intermittently. The combination of paracetamol and caffeine is an useful alternative to NSAIDs: it was compared with placebo in two high-quality studies, showing its superiority over placebo as well as a good tolerability [9, 10]. Such studies, however, were carried out in Anglo-Saxon populations, with different habits respect to Italian patients, including higher consumption of caffeine, and therefore a different sensitivity to its peripheral and central stimulatory effects.

The main aim of this study was to confirm in an Italian population affected by TTH the good profile of safety and tolerability of the combination paracetamol $1000 \mathrm{mg}-$ caffeine $130 \mathrm{mg}$ observed in previous studies [9, 10], by a comparison with naproxen sodium, a NSAID widely used in the acute treatment of TTH [11-13], and placebo. A secondary objective was to assess the efficacy of the combination paracetamol $1000 \mathrm{mg}$-caffeine $130 \mathrm{mg}$ in the acute treatment of TTH, versus naproxen sodium $550 \mathrm{mg}$ and placebo.

\section{Patients and methods}

\section{Patients}

In this multicentre study, conducted between December 2004 and May 2007 in eight Headache Centres throughout Italy, outpatient volunteers of both genders (18-65 years) with a clinical history of TTH were recruited if they fulfiled the following inclusion criteria:

- Diagnosis of episodic tension-type headache according to the ICHD-II criteria [14], modified in the single, following criterion: absence of nausea, vomiting, photophobia and phonophobia (to exclude subjects with migraine headaches),

- Mean frequency of 4-14 days with TTH per month,

- History of response to treatment of TTH with over-thecounter pain-killers,
- Daily consumption of at least two cups of coffee,

- Adequate contraception in women of fertile age,

- Medical history and physical examination inconsistent with organic disorders associated with headaches.

Subjects were excluded from the study if they had a history of any of the following:

- Known hypersensitivity or allergy to paracetamol or naproxen,

- Chronic headache, either recurrent or continuous,

- Concomitant use/overuse of NSAIDs or other analgesics; treatment with antiplatelet or anticoagulant drugs,

- Migraine or post-traumatic headache,

- Alcohol abuse, drug dependency, or psychiatric disease,

- Coagulation disorders, peptic ulcer disease, pancreatic disease, clinically significant renal or hepatic disease, blood hypertension, mild/moderate kidney or liver failure, Gilbert's syndrome.

The study was conducted in accordance with the Declaration of Helsinki (Tokio version 2004) and Good Clinical Practice standards and did not start before independent Ethics Committee approval appropriate for each investigator was obtained. Prior to enrolment the patients gave their written informed consent; they were allowed to terminate participation in the trial at any time, without giving reasons. This trail complies with the Guidelines for trials of drug treatments in TTH of the International Headache Society [15].

\section{Study design and treatments}

This study was designed as a randomised, double-blind, double-dummy, crossover, placebo-controlled trial. After having signed the informed consent form, patients were required to treat the next three consecutive TTH attacks with the investigational study medications, according to a randomised crossover sequence which was computer generated using Microsoft ${ }^{\circledR}$ Access 2003. Each patient was randomly allocated to one of the six sequences illustrated in Table 1, according to a 1:1:1:1:1:1 scheme. Eligible patients were assigned in sequential order of entry. Access to the randomisation code was strictly controlled and the treatment assignment remained unknown to all parties involved in the trial until formal database lock. Subjects in all treatment groups received three identical boxes (numbered progressively from 1 to 3 to indicate the exact order in which they should have been used) containing: one soft gel capsule containing one tablet of placebo and one sachet containing paracetamol 1,000 mg + caffeine $130 \mathrm{mg}$ [Tachicaf $^{\circledR}$, Angelini Farmaceutici, ACRAF S.p.A., Pomezia (RM), Italy] or one soft gel capsule containing one tablet of naproxen sodium $550 \mathrm{mg}$ (Synflex Forte ${ }^{\circledR}$, 
Recordati S.p.A., Milano, Italy) and one sachet of placebo or one soft gel capsule containing one tablet of placebo and one sachet of placebo. The doses corresponded to the maximal individual therapeutic single doses recommended for these analgesics. Blinding was ensured using matched trial supplies, identical in colour, size, shape and taste. At each TTH attack patients would have to take one soft gel capsule and one sachet at the same time. The trial medication was to be taken when the headache occurred, and when the patients would normally have taken their usual analgesic. Other than study medication, patients received rescue medication (ibuprofen $600 \mathrm{mg}$ ), to be taken $2 \mathrm{~h}$ after the administration of the trial medication, if the pain persisted.

At baseline visit, patients' medical histories and concomitant treatments were recorded, vital signs were registered and physical examination was performed by the investigator. Patients were required to record in a headache diary, the date and time of drug ingestion, pain intensity before treatment and pain intensity, pain relief and adverse events (AEs) after treatment recorded at 1, 2, 3, and $4 \mathrm{~h}$. At the end of 4-h measurement interval or at the time of use of rescue medication, patients had to record the presence and intensity of AEs and to evaluate their impression of the efficacy and tolerability of the study medication. The same procedures were repeated for the two subsequent TTH attacks, provided that they were $48 \mathrm{~h}$ apart from each other.

Patients had to contact the investigator within $48 \mathrm{~h}$ after the third treated episode of TTH for the final visit. The investigator reviewed the completed diary with the patient to ensure that all required information had been registered, recorded vital signs and concomitant treatments and expressed a global assessment of tolerability. Patients were asked to indicate their preference for one of the three treatments, taking into account efficacy and tolerability.

\section{Outcomes}

Safety and tolerability were evaluated by comparing vital signs at screening and final visits and by recording AEs. AEs could be recorded by the investigator or by the patient [filling in a symptom check-list (including nervousness, palpitation, insomnia, dizziness, abdominal pain, dyspepsia, nausea, vomiting, drowsiness and fatigue) hourly for $4 \mathrm{~h}$ after the study medication ingestion]. AEs intensity

Table 1 Sequences of study treatments

$P C F$ paracetamol $1,000 \mathrm{mg}+$ caffeine $130 \mathrm{mg}$, $N A P$ naproxen sodium $550 \mathrm{mg}$, $P L A$ placebo was determined by subjective evaluation of the patient and classified as mild (signs or symptoms easily tolerated), moderate (discomfort sufficient to cause interference with normal activities) and severe (incapacitating with inability to do work or undertake normal activity). A global assessment of tolerability was expressed by the patient, using a 5-point Verbal Rating Scale (VRS: 'excellent', 'very good', 'good', 'sufficient' and 'poor'). The investigator expressed a global assessment of tolerability, using the following 5-point VRS: 'very good' (no AEs), 'good' (mild AEs recovered spontaneously without treatment), 'fair' (AEs requiring treatment to recover), 'poor' (AEs requiring withdrawal from study), 'very poor' (serious AEs).

To assess treatments' efficacy, intensity of pain (on a 4-point scale: 0 'absent', 1 'mild', 2 'moderate', 3 'severe') and pain relief (on a 5-point scale: 0 'no relief', 1 'little relief', 2 'some relief', 3 'much relief', 4 'complete relief') were evaluated hourly during the 4-h post-dose period. Based on these two variables, the following parameters were calculated:

- Pain intensity difference (PID). For each patient the sum of pain intensity differences (SPID) was calculated as the sum of differences between pre-dose assessment and every post-dose assessment,

- Total pain relief (TOTPAR), calculated as the sum of every post-dose assessment.

Finally, patients expressed their preference for one of the investigational treatments, answering the following question: "Taking into account both tolerability and efficacy, which of the three treatments would you take again at the next headache attack?" (Options were: (a) none, (b) treatment number 1 , (c) treatment number 2 , (d) treatment number 3).

Statistical analysis

The study was powered to test the primary hypothesis, namely that paracetamol $1,000 \mathrm{mg}+$ caffeine $130 \mathrm{mg}$ (PCF) would be non-inferior to naproxen sodium $550 \mathrm{mg}$ (NAP) as regards the proportion of patients complaining of AEs in the 4-h post-dose period. Assuming a 7\% threshold as the maximum tolerated difference between PCF and NAP, a $7.9 \%$ proportion of discordant pairs, $80 \%$ power, and one-tailed test with a 0.05 significance level, the required sample size was estimated to be 100 treated patients. In order to take any premature withdrawals into account, the number of patients to be enrolled was set at 150.

According to study protocol, three populations have been evaluated: per-protocol (PP, subjects fulfiling all the inclusion criteria who took the three treatments and 
performed all the tolerability evaluations), randomized patients (RP, patients who took at least one of the treatments), and intention-to-treat [ITT, patients who took the three treatments and performed at least one post-dose evaluation. Data missing for any scheduled evaluation was replaced by the last observation carried forward (LOCF) procedure]. The tolerability endpoints were evaluated using PP and RP populations; ITT population was employed for efficacy analyses. Descriptive statistics on RP population was used for demographic and baseline characteristics.

The Mc Nemar test (reported as $90 \%$ confidence interval) was used to compare the percentage of patients who recorded AEs after each treatment. Besides those recorded by patients in the 4-h post-dose period, all the AEs were classified by the investigator on the basis of: treatment received, system involved, severity and correlation with the investigational medication. The analysis of variance was used to evaluate the differences of vital signs respect to baseline. The Wilcoxon signed rank test was used to analyse the global assessment of tolerability expressed by patients.

The analysis of variance was used to evaluate SPID and TOTPAR. The patient' preference for one of the treatments was reported as a distribution of frequency.

The evaluation of the quality and completeness of the data, identification of important protocol deviations and handling of problem cases were performed regularly and finally decided before locking and unblinding the database.

\section{Results}

\section{Study population}

One-hundred and eleven subjects entered the study, 99 of whom took at least one of the treatments (RP). Twelve patients were excluded from the study, since they did not fulfil the inclusion criteria $(n=2)$ or did not take any medication $(n=10)$. Other six patients took 1 or 2 investigational medications only. Therefore PP population included 93 subjects. Reasons why patients did not complete the study were: explicit request to withdraw from the study $(n=8)$, lack of compliance to study procedures $(n=5)$, severe nausea $(n=1)$, unmasking of assigned treatment $(n=1)$, expiry of investigational medication $(n=1)$. ITT population included 93 subjects, 91 and 81 of whom were available for the efficacy analyses concerning pain severity and pain relief, respectively.

Demographic characteristics and headache history of RP population are shown in Table 2. As regards baseline pain intensity of treated headaches $(n=287), 47$ attacks (16.4\%) were judged to be mild, 168 (58.5\%) moderate and $72(25.1 \%)$ severe.
Table 2 Demographic characteristics and headache history (randomized patients dataset; $n=99$ )

\begin{tabular}{lc}
\hline Gender, $n(\%)$ & $40(40.4)$ \\
Male & $59(59.6)$ \\
Female & \\
Age, years & $35.1 \pm 10.19$ \\
Mean \pm SD & $19-64$ \\
Range & \\
Race, $n(\%):$ & $98(99.0)$ \\
Caucasian & $1(1.0)$ \\
Other & $22.2 \pm 9.09$ \\
Headache duration, years & $6-44$ \\
Mean \pm SD & $2(2.0)$ \\
Range & $97(98.0)$ \\
Mean number of days with tension-type headache per month, $n(\%)$ \\
$<4$ \\
$4-14$ \\
$>14$
\end{tabular}

Tolerability and safety

Considering the PP population $(n=93)$, in which all the tolerability assessments were available, the following percentages of patients reported AEs in the 4-h post-dose period: $36.6 \%$ with PCF, $31.2 \%$ with NAP, and $36.6 \%$ with placebo (PLA). Comparing PCF and NAP, the proportion of discordant pairs (that is, patients who reported AEs with one but not the other drug and vice versa) were 19.4 and $14.0 \%$, respectively, with a difference of $5.4 \%(90 \% \mathrm{CI},-4.4$ to $15.2 \%)$. Comparing PCF and PLA, the proportion of discordant pairs was $14.0 \%$ for both treatments, with no differences of discordant pairs $(90 \% \mathrm{CI},-9.0$ to $9.0 \%)$. Since in the comparisons PCF-NAP and PCF-PLA the confidence interval included both $\Delta$ (the maximum tolerated difference between treatments) and zero, the difference was nonsignificant but the result regarding noninferiority was inconclusive [16]. Comparing NAP and PLA, the proportion of discordant pairs were 12.9 and $18.3 \%$, respectively, with a difference of $-5.4 \%$ (90\% CI, -14.9 to $4.1 \%$ ). Therefore NAP was noninferior to PLA. During the 4-h post-dose period, 224 AEs were recorded in the RP population, 76 (33.9\%) after PCF ingestion, 66 (29.5\%) after NAP and 82 (36.6\%) after PLA. Table 3 illustrates the percentage of AEs according to investigational drugs. Most AEs were codified as mild or moderate. Only 21 AEs $(9.4 \%)$ were recorded as severe: $13(5.8 \%)$ after PLA, $5(2.3 \%)$ after NAP and 3 (1.3\%) after PCF. The most frequently observed AEs were nausea, drowsiness, fatigue and nervousness (Table 3). 
Table 3 Adverse events recorded in 4-h period after ingestion of investigational drugs (randomized patients dataset; $n=99$ )

\begin{tabular}{lccc}
\hline Adverse event & PCF $n(\%)$ & NAP $n(\%)$ & PLA $n(\%)$ \\
\hline Nervousness & $11(14.5)$ & $8(12.1)$ & $12(14.6)$ \\
Palpitation & $4(5.3)$ & $2(3.0)$ & $3(3.7)$ \\
Insomnia & $4(5.3)$ & $3(4.6)$ & $3(3.7)$ \\
Dizziness & $6(7.9)$ & - & $4(4.9)$ \\
Abdominal pain & $5(6.6)$ & $4(6.1)$ & $5(6.1)$ \\
Dyspepsia & $6(7.9)$ & $7(10.6)$ & $5(6.1)$ \\
Nausea & $15(19.7)$ & $15(22.7)$ & $21(25.6)$ \\
Vomiting & $2(2.6)$ & $3(4.6)$ & $2(2.4)$ \\
Drowsiness & $11(14.5)$ & $14(21.2)$ & $13(15.9)$ \\
Fatigue & $12(15.8)$ & $10(15.2)$ & $14(17.1)$ \\
\hline
\end{tabular}

PCF paracetamol $1,000 \mathrm{mg}+$ caffeine $130 \mathrm{mg}(n=98), \quad N A P$ naproxen sodium $550 \mathrm{mg}(n=94), P L A$ placebo $(n=98)$

Besides AEs directly recorded by the patients, other 20 AEs were registered by the investigator. Only two of them (sweating and lips dryness), however, were judged to be possibly correlated to the drug. No serious AEs occurred. One patient dropped from the study because of severe nausea during a headache attack treated with PCF. No significant differences occurred in vital signs recorded at final visit compared to those recorded at screening visit.

The global assessment of tolerability expressed by the patient is shown in Table 4. The evaluation was 'excellent' or 'very good' in $45.7 \%$ of the patients after PCF, in $51.6 \%$ after NAP, and $41.7 \%$ after PLA. The difference was statistically significant $(P<0.05)$ between NAP and PLA.

The global assessment of tolerability expressed by the investigator on the RP population $(n=97)$ at the final visit and referring to the sum of investigational drugs, gave the following results: 'very good' or 'good' in $96 \%$, 'fair' in $3 \%$ and 'poor' in $1 \%$ of the patients.

\section{Efficacy}

Figure 1 reports the time course of PID for the three investigational treatments, whilst the SPID mean values

Table 4 Global assessment of tolerability expressed by the patient (randomized patients dataset)

\begin{tabular}{llll}
\hline & PCF $n(\%)$ & NAP $n(\%)$ & PLA $n(\%)$ \\
\hline Excellent & $17(18.1)$ & $32(35.2)$ & $20(20.8)$ \\
Very good & $26(27.7)$ & $15(16.5)$ & $20(20.8)$ \\
Good & $26(27.7)$ & $23(25.3)$ & $22(22.9)$ \\
Sufficient & $12(12.8)$ & $9(9.9)$ & $14(14.6)$ \\
Poor & $13(13.8)$ & $12(13.2)$ & $20(20.8)$ \\
Total & $94(100.0)$ & $91(100.0)$ & $96(100.0)$ \\
\hline
\end{tabular}

$P C F$ paracetamol $1,000 \mathrm{mg}+$ caffeine $130 \mathrm{mg}$, NAP naproxen sodium $550 \mathrm{mg}, P L A$ placebo

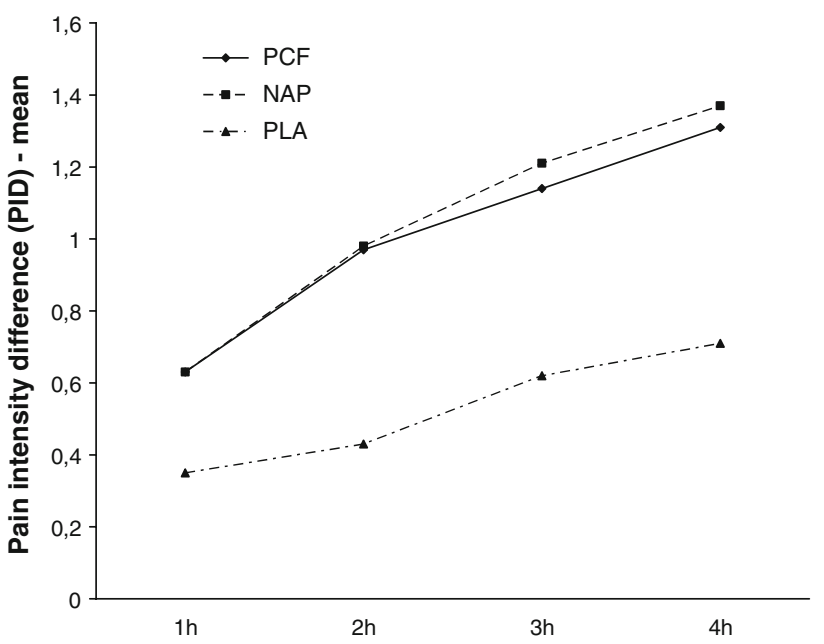

Fig. 1 Time course of the mean pain intensity difference (PID) for the three investigational treatments (intention-to-treat dataset, $n=91) . \quad P C F$ paracetamol $1,000 \mathrm{mg}+$ caffeine $130 \mathrm{mg} ; \quad N A P$ naproxen sodium $550 \mathrm{mg}$, PLA placebo

with standard errors are illustrated in Table 5. PID relative to baseline steadily increased over time in both the active treatments compared to placebo. PCF and NAP were significantly better than placebo $(P<0.05)$, whilst no differences emerged between the two active treatments.

The time course of TOTPAR during the 4-h observation period is shown in Fig. 2, whilst TOTPAR mean values with standard errors are displayed in Table 5. Both PCF and NAP provided significantly more relief than placebo $(P<0.05)$, but were not significantly different from each other.

The percentage of subjects using rescue medication was similar for PCF and NAP (4.8 and 3.3\%, respectively) and both were less than the $10.0 \%$ of subjects who used rescue medication after PLA.

As regards the patients' preference for one of the tested treatments (RP dataset, $n=92$ ), 30 patients (32.6\%) preferred PCF, 41 patients (44.6\%) NAP and 21 patients (22.8\%) PLA.

Table 5 Sum of pain intensity differences (SPID) and total pain relief (TOTPAR) in the 4-h observation period (intention-to-treat dataset)

\begin{tabular}{llll}
\hline & PCF & NAP & PLA \\
\hline SPID & & & \\
$n$ & 91 & 91 & 91 \\
Baseline intensity (SE) & $2.0(0.06)$ & $2.1(0.07)$ & $2.1(0.08)$ \\
Mean (SE) & $4.0(0.35)$ & $4.2(0.38)$ & $2.1(0.35)$ \\
TOTPAR & & & \\
$n$ & 81 & 81 & 81 \\
Mean (SE) & $8.5(0.60)$ & $8.0(0.63)$ & $4.8(0.60)$ \\
\hline
\end{tabular}

PCF paracetamol $1,000 \mathrm{mg}+$ caffeine $130 \mathrm{mg}$, NAP naproxen sodium $550 \mathrm{mg}$, PLA placebo 


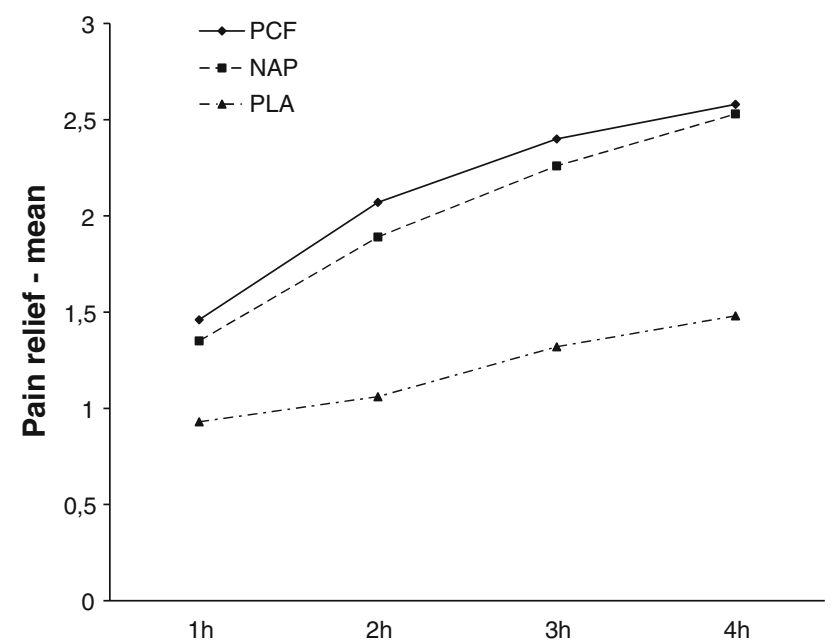

Fig. 2 Time course of total pain relief (TOTPAR) for the three investigational treatments (intention-to-treat dataset, $n=81$ ). $P C F$ paracetamol $1,000 \mathrm{mg}+$ caffeine $130 \mathrm{mg}, N A P$ naproxen sodium $550 \mathrm{mg}$, PLA placebo

\section{Discussion}

In a condition as widespread as TTH is, using the most effective dose of a drug that is well tolerated by a patient (and safe) is a reasonable basis for selecting a medication. Exerting its analgesic activity thanks to a direct effect on the central nervous system [17], at least in part mediated by the serotonergic system [18, 19], paracetamol has some advantages over NSAIDs, due to its scarce inhibition of peripheral cyclooxygenase: it is well tolerated at gastrointestinal level, it is only a weak inhibitor of aggregation of platelets and does not alter the bleeding time [20]. In a recent systematic review on the therapy of acute episodic TTH [7], paracetamol 1,000 mg was significantly more effective than placebo with AEs matching those of placebo. A quantitative analysis of seven studies comparing NSAIDs and paracetamol, however, showed a significant difference in short-term pain relief in favour of NSAIDs [7]. The combination paracetamol $1,000 \mathrm{mg}$ and caffeine $130 \mathrm{mg}$ has been proved to be superior both to placebo and paracetamol $1,000 \mathrm{mg}$ in the treatment of TTH [10], at no significant cost in term of AEs, thanks to the synergistic effect of caffeine. Caffeine has a direct analgesic effect not mediated via effects on mood or on caffeine withdrawal [9]. Suggested, albeit unproven, mechanisms for caffeine analgesia are the antagonistic activity on adenosine receptors [21] and the ability to increase the norepinephrine activity in the central nervous system [22]. Moreover, caffeine has a well-documented adjuvant action, when combined with analgesics [23]. The mechanisms of this analgesic adjuvant effect are not well-established, but could, at least partially, be explained by pharmacokinetic interactions, in terms of increased absorption or reduced metabolism of analgesics induced by caffeine [24, 25]. Whatever the mechanisms are, it has been shown that the addition of caffeine to a NSAID increases its analgesic potency by $40 \%$ [26].

Some worry as far as Italy is concerned could be raised by the fact that the studies on the 'high-dose' paracetamolcaffeine combination (i.e., paracetamol 1,000 mg and caffeine $130 \mathrm{mg}$ ) were carried out in Anglo-Saxon populations, with a consumption of caffeine higher than that found in Italians, and therefore theoretically less prone to the stimulating effects of caffeine. The results of the present study are absolutely reassuring about the tolerability of the paracetamol-caffeine combination. The difference amongst treatments was nonsignificant, even though the noninferiority of PCF compared to NAP and PLA could not be conclusively demonstrated. Moreover, the percentage of patients complaining of AEs in the 4-h post-treatment period was exactly the same $(36.6 \%)$ after PCF and after PLA. The AEs theoretically attributable to the stimulating effect of caffeine (nervousness, palpitation and insomnia) were roughly the same with the three investigational medications (Table 3). The high frequency of some, even severe, AEs like nausea after PLA is likely to be due to symptoms accompanying headache not extinguished by the active drugs. It is not a chance that a good evaluation in the global assessment of tolerability expressed by the patient was lower after PLA than after active drugs.

As regards the efficacy, this study confirms the good results obtained in previous clinical trials [9, 10], showing a significant superiority of PCF over placebo, and no difference compared to a reference drug as NAP, in both the reduction of pain intensity and the pain relief.

The results of this trial may have important implications for clinical practice. Many drugs for the treatment of TTH are expensive and/or have contraindications or relevant side effects [27]. The combination of paracetamol $1,000 \mathrm{mg}$ and caffeine $130 \mathrm{mg}$ seems to be effective and well tolerated, in particular not showing the specific stimulatory effects of caffeine. It is therefore a serious candidate for the first line treatment of acute episodic TTH.

Acknowledgments This work was supported by a grant from Angelini Farmaceutici, ACRAF SpA (Rome, Italy).

Conflict of interest The study was sponsored by Angelini Farmaceutici, ACRAF SpA. Two of the authors (G. Di Loreto, P. Dioniso) are Angelini employees.

\section{References}

1. Stovner L, Hagen K, Jensen R et al (2007) The global burden of headache: a documentation of headache prevalence and disability worldwide. Cephalalgia 27:193-210 
2. Lyngberg AC, Rasmussen BK, Jørgensen T et al (2005) Has the prevalence of migraine and tension-type headache changed over a 12-year period? A Danish population survey. Eur J Epidemiol 20:243-249

3. Rasmussen BK, Jensen R, Olesen J (1992) Impact of headache on sickness absence and utilisation of medical services: a Danish population study. J Epidemiol Community Health 46:443-446

4. Lyngberg AC, Rasmussen BK, Jensen R et al (2005) Secular changes in health care utilization and work absence for migraine and tension-type headache. A population based study. J Epidemiol Com Health 20:1007-1014

5. Schwartz BS, Stewart WF, Simon D et al (1998) Epidemiology of tension-type headache. JAMA 279:381-383

6. Lenaerts ME (2006) Burden of tension-type headache. Curr Pain Headache Rep 10:459-466

7. Verhagen AP, Damen L, Berger MY (2006) Is any one analgesic superior for episodic tension-type headache? J Fam Pract 55:1064-1072

8. Tfelt-Hansen P (2007) Acute pharmacotherapy of migraine, tension-type headache, and cluster headache. J Headache Pain 8:127-134

9. Ward N, Whitney C, Avery D et al (1991) The analgesic effects of caffeine in headache. Pain 44:151-155

10. Migliardi JR, Armellino JJ, Friedman M et al (1994) Caffeine as an analgesic adjuvant in tension headache. Clin Pharmacol Ther 56:576-586

11. Miller DS, Talbot CA, Simpson W et al (1987) A comparison of naproxen sodium, acetaminophen and placebo in the treatment of muscle contraction headache. Headache 27:392-396

12. Lange R, Lentz R (1995) Comparison ketoproten, ibuprofen and naproxen sodium in the treatment of tension-type headache. Drugs Exp Clin Res 21:89-96

13. Prior MJ, Cooper KM, May LG et al (2002) Efficacy and safety of acetaminophen and naproxen in the treatment of tension-type headache. A randomized, double-blind, placebo-controlled trial. Cephalalgia 22:740-748

14. Headache Classification Subcommittee of the International Headache Society (2004) The International Classification of Headache Disorders, 2nd edn. Cephalalgia 24(Suppl 1):1-160
15. International Headache Society Committee on Clinical Trials (1995) Guidelines for trials of drug treatments in tension-type headache (1st edn). Cephalalgia 15:165-179

16. Piaggio G, Elbourne DR, Altman DG et al (2006) Reporting of noninferiority and equivalence randomized trials. An extension of the CONSORT statement. JAMA 295:1152-1160

17. Piletta P, Porchet HC, Dayer P (1991) Central analgesic effect of acetaminophen but not of aspirin. Clin Pharmacol Ther 49: 350-354

18. Pini LA, Vitale G, Ottani A et al (1997) Naloxone-reversible antinociception by paracetamol in the rat. J Pharmacol Exp Ther 280:934-940

19. Sandrini M, Romualdi P, Capobianco A et al (2001) The effect of paracetamol on nociception and dynorphin A levels in the rat brain. Neuropeptides 35:110-116

20. Botting RM (2000) Mechanism of action of acetaminophen: is there a cyclooxygenase 3? Clin Infect Dis 31(suppl 5):S202-S210

21. Daly JW, Bruns RF, Snyder SH (1981) Adenosine receptors in the central nervous system: relationship to the central actions of methylxanthines. Life Sci 28:2083-2097

22. Galloway MP, Roth RH (1983) Neuropharmacology of 3-isobutylmethylxanthine: effects on central noradrenergic systems in vivo. J Pharmacol Exp Ther 227:1-8

23. Sawynok J, Yaksh TL (1993) Caffeine as an analgesic adjuvant: a review of pharmacology and mechanisms of action. Pharmacol Rev 45:43-85

24. Onrot J, Shaheen O, Biaggioni I et al (1986) Reduction of liver plasma flow by caffeine and theophylline. Clin Pharmacol Ther 40:506-510

25. Iqbal N, Ahmad B, Janbaz KH et al (1995) The effect of caffeine on the pharmacokinetics of acetaminophen in man. Biopharm Drug Dispos 16:481-487

26. Laska EM, Sunshine A, Mueller F et al (1984) Caffeine as an analgesic adjuvant. JAMA 251:1711-1718

27. Schoenen J, Wang W (1997) Tension-type headache. In: Goadsby PJ, Silberstein SD (eds) Headache. Butterworth-Heinemann, Boston, pp 177-200 